\title{
VERSATILE RP-HPLC METHOD DEVELOPMENT FOR QUANTITATIVE ESTIMATION OF TELMISARTAN AND RAMIPRIL IN ANIMAL PLASMA
}

\author{
PRANAY WAL*, RUCHI TIWARI, ANKITA WAL, GAURAV TIWARI
}

Pranveer Singh Institute of Technology, Kanpur-Agra-Delhi National Highway-2, Bhauti, Kanpur, India Email: pranaywal@gmail.com

Received: 29 Nov 2017, Revised and Accepted: 24 May 2018

\begin{abstract}
Objective: A fast, specific, and sensitive high-performance liquid chromatographic method has been developed and validated for the quantitative determination of unchanged Ramipril (RAM) and Telmisartan (TEL) in animal plasma.

Methods: Analytes were extracted from animal plasma, $250 \mu \mathrm{l}$ of animal plasma sample were mixed with internal working standard $\left(25 \mathrm{ngmL}^{-1}\right)$ with the further addition of chloroform (HPLC Grade, Merck). The clear organic layer was separated and reconstituted to 1 ml in mobile phase and analysed by HPLC. The method was validated and evaluated in terms of linearity, accuracy, precision, specificity, limit of detection and limit of quantitation.

Results: Absorption maxima of TEL and RAM was found to be 270 and $273 \mathrm{~nm}$ respectively. TEL and RAM with their respective internal standards (I. S.) were found to be well separated from the co-eluted components and there were no interferences from the endogenous material. The limit of detection (LOD) and limit of quantitation (LOQ) were found to be $2.01 \pm .05 ; 4.88 \pm 0.10$ and 0.11 and 0.25 for TEL and RAM respectively on the basis of a signal to noise ratio. The ruggedness of the method at various parameters was found to be $\pm 1.94 \%$ and $\pm 1.02 \%$ for TEL and RAM respectively. The low values of \%RSD $(<2.0)$ for each of the drug proposed that during all deliberate variations, middle-quality control (MQC) was not affected and it was in accordance with that of actual.
\end{abstract}

Conclusion: Thus developed High-Performance Liquid Chromatography (HPLC) method was found to be more accurate, precise, sensitive, selective and reproducible.

Keywords: Ramipril, Telmisartan, Reverse Phase High-Performance Liquid Chromatography (RP-HPLC) and Validation

(C) 2018 The Authors. Published by Innovare Academic Sciences Pvt Ltd. This is an open access article under the CC BY license (http://creativecommons.org/licenses/by/4.0/) DOI: http://dx.doi.org/10.22159/ijap.2018v10i4.24023

\section{INTRODUCTION}

Telmisartan (TEL) and Ramipril (RAM) are a safe and effective alternative for the treatment of hypertension. Moreover, due to its good tolerability, an increasing use of TEL in cardiovascular high risk animals can be anticipated. RAM is long-acting angiotensinconverting-enzyme (ACE) inhibitor and known for its extensive tissue distribution. Chemically RAM is $(1 S, 5 S, 7 S)-8-((2 S)-2-(((1 S)-1-$ ethoxycarbonyl-3-phenyl-propyl)amino) propanoyl)-8-azabicyclo (3.3.0) octane-7-carboxylic acid (fig. 1). RAM is a prodrug and nonsulfhydryl angiotensin-converting enzyme (ACE) inhibitor with antihypertensive activity [1]. RAM is converted in the liver by deesterification into its active form ramiprilat, which inhibits ACE, thereby blocking the conversion of angiotensin I to angiotensin II. This abolishes the potent vasoconstrictive actions of angiotensin II and leads to vasodilatation. This agent also causes an increase in bradykinin levels and a decrease in angiotensin II-induced aldosterone secretion by the adrenal cortex, thereby promoting diuresis and natriuresis. TEL chemically 4'-[(1, 4'-Dimethyl-2'-propyl-[2, 6'-bi-1Hbenzimidazol]-1'-yl) methyl]-[1, 1'-biphenyl]-2-carboxylic acid (fig. 2), is a nonpeptide angiotensin-II receptor antagonist, which selectively and insurmountably inhibits angiotensin-II AT1 receptor subtype without affecting other systems involved in cardiovascular regulation [1]. TEL is an angiotensin receptor blocker (also called an angiotensinII receptor antagonist (AIIRA)). It is used to treat hypertension (high blood pressure). People with high blood pressure often do not feel unwell but, left untreated, high blood pressure can harm the heart and damage blood vessels. TEL work by blocking the effect of a chemical called angiotensin II found in bloodstream. Angiotensin II causes blood vessels to narrow, so by blocking this effect. TEL allows blood vessels to relax and widen. As this happens, the pressure within blood vessels is reduced. This also makes it easier for the heart to pump blood around Body. TEL is also used to help prevent heart attacks and strokes in people who may be at risk of these because of other medical conditions, such as diabetes. Literature survey revealed that telmisartan is not yet official in any of the pharmacopoeia. RAM is official in United States of pharmacopoeia (USP) and British
Pharmacopoeia (BP) where high pressure liquid chromatography (HPLC) and potentiometric titration is the official method of analysis. There are numerous methods reported for estimation of these drugs alone as well as in combination with other drugs in pharmaceutical dosage forms [2-5] and/or in biological fluids. However, no method has been reported so far in the estimation of these two drugs simultaneously in combined dosage forms. A new RP-HPLC method for simultaneous estimation of TEL and RAM also has been developed. Although RP-HPLC method for simultaneous estimation of TEL and RAM and spectrophotometric method for estimation of TEL and RAM individual and in combination with other drug method has been developed to quantify TEL and RAM but no Spectrophotometric method has been developed for simultaneous estimation of TEL and RAM in combined dosage form.

The aim of this study was to establish a simple, rapid, economic and accurate RP-HPLC method for the identification and measurement of TEL and RAM in plasma correlating the plasma concentration with dose and clinical picture of animals; beneficial to certain group of the population. The assay requires a small sample volume, involves a single step liquid-liquid extraction with a specific internal standard and a short chromatographic run.

\section{MATERIALS AND METHODS}

\section{Chemicals and reagents}

TEL (fig. 1) and RAM (fig. 2) were gifted by Ipca Pharmaceutical Pvt. Ltd, Ratlam (Madhya Pradesh). All reagents used were of HPLC grade except acetic acid which was of analytical grade. Water was glass triple-distilled and further purified with a $0.44 \mu$ filtration membranes using a vacuum pump.

\section{Equipments}

The HPLC system used consisted of Shimadzu LC-2010 AHT/ 2010Cht, Kyoto, Japan, solvent delivery system, a system controller, an UV-Visible detector (PCB, LC2K-UV1) operated at wavelength 280 $\mathrm{nm}$, a degasser and data processor all of SHIMADZU. 
<smiles>CCC[C@@H]1CC[C@H](C(=O)O)N1C(=O)[C@H](C)N[C@@H](CCc1ccccc1)C(=O)OCC</smiles>

Fig. 1: Structure of RAM

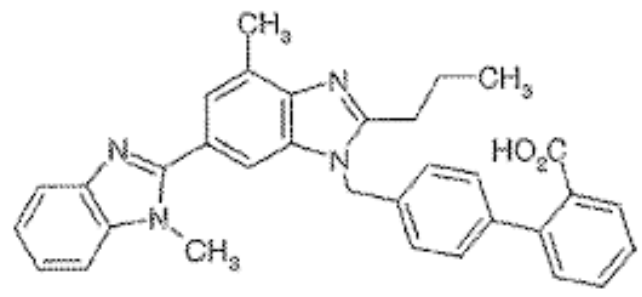

Telmisartan

Fig. 2: Structure of TEL

\section{Preparation of standard solutions of TEL}

One mg of pure TEL was weighed and transferred into a round bottom flask, $100 \mathrm{ml}$ of methanol was added and sonicated for 15 min for complete dissolution of the drug. Now, the solution was filtered through a $0.45 \mu \mathrm{m}$ pore size nylon 66 membrane filters using a vacuum pump and further diluted using methanol to give stock solutions $10 \mu \mathrm{g} / \mathrm{ml}$ and stored at $4{ }^{\circ} \mathrm{C}$.

\section{Method development for TEL}

Based on the solubility profile, pKa, polarity of a drug molecule and other factors like stability e. t. c, various mobile phase combination, flow rate, $\mathrm{pH}$ and $\lambda_{\max }$ was selected and samples were run for optimum selection of chromatographic parameters to develop and validate the RP-HPLC method for estimation of TEL in biological matrices like plasma. The final set of chromatographic conditions was established using C18-ODS column (Thermo), $250 \mathrm{~mm} \times 4.6 \mathrm{~mm}$ I.D., $5 \mu \mathrm{m}$ particle sizes which were protected by a guard column $(1$ $\mathrm{cm} \times 4.0 \mathrm{~mm}$ I.D., $5 \mu \mathrm{m}$ particle size). A mixture of methanol: Acetonitrile (70: 30 ) containing $0.1 \%$ Acetic Acid (pH adjusted to 2.5 using $0.05 \mathrm{M} \mathrm{KHPO}_{4}$ ) was used as the mobile phase (isocratic separation). The detection wavelength was $270 \mathrm{~nm}$ and injection volume $10 \mu \mathrm{l}$. The mobile phase was filtered, degassed and pumped at a flow rate of $1.2 \mathrm{ml} / \mathrm{min}$.

\section{Extraction of TEL from animal plasma}

Pharmacokinetic studies were carried out using healthy male Wistar rats weighing $240-270 \mathrm{~g}$. The animals were housed in polyacrylic cages and maintained under standard laboratory conditions (temperature $25 \pm 2{ }^{\circ} \mathrm{C}$ ) with dark and light cycles for at least seven days prior to the experiment and were given a commercial rat chow and water ad libitum. The experimental protocol was approved (IAEC/PSIT/1273/ac/2017) by the Institutional Animal Ethical Committee prior to the conduct of the animal experiments. After an overnight fast, the rats were given an oral administration of a mixture of RAM $(1 \mathrm{mg} / \mathrm{kg})$, and TEL $(1 \mathrm{mg} / \mathrm{kg})$ dissolved in $0.1 \%$ carboxymethylcellulose. The animals had free access to water after $4 \mathrm{~h}$ of the oral administration of drugs. The blood $(\sim 250 \mu \mathrm{l})$ was collected into heparinized tubes from the suborbital veniplex before administration and at $0.08,0.15,0.5,1.0,1.5,2.0,3.0,4.0,6.0,8.0,12.0$, $24.0,36.0,48.0$, and $72.0 \mathrm{~h}$ after dosing. The plasma was immediately separated by centrifugation and stored frozen at $-80^{\circ} \mathrm{C}$ until analysis. $250 \mu \mathrm{l}$ of animal plasma sample was mixed with $50 \mu \mathrm{l}$ of I. S. working standard $(25 \mathrm{ng} / \mathrm{ml})$ and are vortex-mixed for $5 \mathrm{~min}$. Then $300 \mu \mathrm{l}$ of chloroform [selected after extensive hit and trial extractions from solvents of different polarities] (HPLC Grade, Merck) was added and centrifuged at 10,000 rpm for $5 \mathrm{~min}$. The clear organic layer was separated and reconstituted to $1 \mathrm{ml}$ in the mobile phase, $100 \mu \mathrm{l}$ was injected in the loop of Rheodyne valve for HPLC analysis.

\section{Preparation of stock solutions and working standards of RAM}

Various mobile phase combination, flow rate, $\mathrm{pH}$ and $\lambda_{\max }$ was selected and samples were run for optimum selection of chromatographic parameters to develop and validate RP-HPLC method (as per the Food and Drug Administration (FDA) guidelines for bioanalytical method development and validation) for estimation of RAM in a biological matrix like plasma. The RP-HPLC method was developed and validated, eluents were monitored by absorbance at $273 \mathrm{~nm}$ using a mixture of methanol: water (pH adjusted to $4.5 \mathrm{using}$ dilute orthophosphoric acid) in the ratio of $72: 28(\mathrm{v} / \mathrm{v})$ at a flow rate of $1.0 \mathrm{ml} / \mathrm{min}$ with C18 column. The detection wavelength was 273 $\mathrm{nm}$ which was selected by analyzing overlain UV spectra of RAM of concentration $10 \mu \mathrm{g} / \mathrm{ml}$ each.

\section{Extraction of RAM from animal plasma}

\section{Preparation of the working standard stock solution}

A primary stock solution $(1 \mathrm{mg} / \mathrm{ml})$ was prepared by dissolving 10 $\mathrm{mg}$ of RAM in $10 \mathrm{ml}$ of HPLC grade methanol. The stock solution was suitably diluted with HPLC grade methanol to obtain a working range of standard solutions. Plasma used in the study was isolated from blood by centrifugation at $10000 \mathrm{rpm}$ for a period of $15 \mathrm{~min}$ at $4{ }^{\circ} \mathrm{C}$, using a centrifuge.

\section{Preparation of the working standard stock solution from plasma}

In $5 \mathrm{ml}$ of a blood sample, $20 \mathrm{mg}$ RAM and $10 \%$ of $0.2 \mathrm{ml}$ trichloroacetic acid were added. The sample was centrifuged at $3000 \mathrm{rpm}$ for $35 \mathrm{~min}$. To settle red blood cells at the bottom. The supernatant was separated and volume was made up to $10 \mathrm{ml}$ to get the solution $1000 \mu \mathrm{g} / \mathrm{ml}$ of each drug. This solution was filtered through syringe filter of size $0.20 \mu \mathrm{m}$. These solutions were diluted with mobile phase to get $200 \mu \mathrm{g} / \mathrm{ml}$ [RAM-100] stock solutions.

\section{Quality samples}

The quality control (QC) samples were prepared by pipetted appropriate aliquots from the standard stock solution into three 10 $\mathrm{ml}$ volumetric flasks. The volume was made up to mark with the mobile phase to get concentrations of 1,2 and $3 \mu \mathrm{g} / \mathrm{ml}$. All samples were stored at refrigerated cold conditions $\left(2-8^{\circ} \mathrm{C}\right)$ and equilibrated to room temperature prior to use.

\section{Preparation of mobile phase}

For preparing a mobile phase, HPLC grade acetonitrile was filtered through a $0.20 \mu \mathrm{m}$ membrane filter and subjected to degas in an ultrasonic bath for a period of $15 \mathrm{~min}$ and then $0.02 \mathrm{M}$ Potassium dihydrogen phosphate $(\mathrm{pH} 3.2)[80: 20 \% \mathrm{~V} / \mathrm{V}]$ was added.

\section{Dilutions}

Different concentrations of $0.5-5 \mu \mathrm{g} / \mathrm{ml}$ were prepared from the stock solution of the working standard. All the dilutions were prepared in Methanol.

\section{Preparation of sample stock solution}

Prior to sample analysis, $100 \mu \mathrm{l}$ of each solution was extracted using $300 \mu \mathrm{l}$ of diethyl ether: dichloromethane $(60: 40 \% \mathrm{v} / \mathrm{v})$ for protein precipitation. Further, each mixture was vortexes for a period of 5 min in a vortex mixer with subsequent centrifugation at $10000 \mathrm{rpm}$, for a period of $10 \mathrm{~min}$ at $4{ }^{\circ} \mathrm{C}$ using a centrifuge. For each sample, an aliquot of a supernatant was isolated and subjected to dry. The residue was reconstituted in $100 \mu \mathrm{l}$ of mobile phase and subsequently centrifuged at $10000 \mathrm{rpm}$ for $10 \mathrm{~min}$ at $4{ }^{\circ} \mathrm{C}$ in a centrifuge. The supernatant was finally collected and directly injected into the HPLC system. This procedure was followed for all samples of the calibration curve and quality control (QC).

\section{Preparation of sample solution for analysis}

To plot the calibrations curve, different concentrations were prepared from the working stock solution individually of 
concentration range $0.4-25.6 \mu \mathrm{g} / \mathrm{ml}$ for RAM and $5-250 \mathrm{ng} / \mathrm{ml}$ for TEL.

\section{Spiking of RAM and TEL in plasma for plasma calibration curve}

Drug-free plasma lots were removed from the deep freezer and allowed to attain room temperature. They were vortexes adequately before pipetting.

\section{Preparation of blank plasma samples}

Plasma isolated from rabbit blood by centrifugation at $10000 \mathrm{rpm}$ for a period of $15 \mathrm{~min}$ at $4{ }^{\circ} \mathrm{C}$, and then spiked $500 \mu \mathrm{l}$ of blood plasma with $100 \mu \mathrm{L}$ of dilutent methanol.

\section{Extraction technique}

\section{Pretreatment of biological samples}

In bioanalysis, the method development step additionally requires the extraction trial in order to recover the analyte and internal standard from the highly complicated biological matrix. One should have knowledge about the nature of the drug, molecular weight, pKa, solubility, ionic character, partition coefficient. In initial stages of method development, our focus was to achieve the limit of quantitation (LOQ) level with precision and accuracy and to check for interference at the retention time of the analyte and internal standard.

\section{Validation parameters}

\section{Linearity and range}

The linearity and range of the calibration curve were evaluated with nine calibration standards containing different concentrations of the respective drug $(0.5-5 \mu \mathrm{g} / \mathrm{ml})$. The study was repeated in triplicates to confirm reproducibility of results. The concentrations of the test samples were back-calculated using linear regression analysis.

\section{Selectivity}

Selectivity of developed method was assessed by comparing chromatograms of three different batches of blank plasma obtained from three individual rabbits with those of corresponding standard plasma samples.

\section{Accuracy and precision}

Intra-day precision and accuracy of the developed method were determined by analyzing six replicates of $\mathrm{QC}$ samples at three concentrations in a single sequence. Similarly, for inter-day precision and accuracy; six replicates QC samples at three concentrations were analyzed on three consecutive days. Accuracy of the method was determined by calculating percentage relative error (\% RE) whereas the precision was determined by calculating percentage relative standard deviation (\% RSD).

\section{Robustness}

The robustness of the developed method was studied by evaluating the effect of small but deliberate variations in chromatographic conditions. The parameters studied were flow rate and mobile phase composition.

\section{Recovery (extraction efficiency)}

To investigate extraction efficiency of the developed method, a set of samples $(n=6)$ at each $Q C$ level was prepared by spiking drug into plasma samples and processed further (pre-extraction). Similarly, the second set of plasma samples was processed first and spiked post extraction at each QC levels. Extraction recovery for each analyte was determined by calculating the ratios of peak areas of the pre-extraction samples to those of the samples of post-extraction.

\section{Limits of detection (LOD) and limit of quantitation (LOQ)}

LOD and LOQ of the developed method were estimated on the basis of standard deviation and slope of the calibration curves.

\section{Method validation}

The proposed method was validated (using plasma matrix) as per FDA guidelines for bioanalytical method validation.

\section{Specificity and selectivity}

Specificity of the method was determined by analyzing six replicates of blank animal plasma obtained from six different sources. Each blank sample was tested for interference, and selectivity was ensured at the lower limit of quantification [LLQ]. The other possible interfering substances like co-administered drugs, blood components like hemoglobin etc, metabolites and excipients were also tested and it was found that no endogenous/external substances interfere with the assay.

\section{Accuracy and precision}

The accuracy and precision of the method can be determined by analyzing the spiked control samples with analyte concentrations around the lower limit of quantitation (LLQ), 2-5 times the LLQ, 0.5 times the upper limit of quantitation (ULQ), ULQ and above ULQ. For acceptance criteria, accuracy should be within $85-115 \%$ of nominal concentration and for precision, the coefficient of variation $(\% \mathrm{CV})$ values should be $<15 \%$ over the calibration range, except at the LLQ, where accuracy should be between $80-120 \%$ and $\%$ CV should not be more than $20 \%$. Choosing three concentrations from above range, each spiked sample was analyzed at a minimum of 6 replicates for within run and between run accuracy and precision. The concentrations selected were 3,10 and $30 \mathrm{ng} / \mathrm{ml}$ for plasma matrix. The results of accuracy and precision have been tabulated in results and discussion.

\section{Recovery}

Recovery experiments were performed by comparing the analytical results for extracted samples low. Medium and high concentration with un-extracted standards injected directly that represents $100 \%$ recovery. Each observation was determined in triplicate. The concentrations selected were 1,2 and $3 \mu \mathrm{g} / \mathrm{ml}$ for plasma matrix. Recovery of internal standard (I. S.) was evaluated by comparing the mean peak areas of extracted samples to mean peak areas of reference solutions (un-extracted) of the same concentration $(3 \mu \mathrm{g} / \mathrm{ml})$.

\section{Linearity, the range of calibration curve}

Calibration curve was prepared in the same biological matrix as the samples in the intended study by spiking the matrix with known concentrations of the analyte including a blank sample (matrix sample without internal standard), a zero sample (matrix sample with internal standard), and six to eight non-zero samples covering the expected range, including LLQ.

\section{LOD and LLQ}

The LOD is the lowest concentration of an analyte in the sample that can be detected but not quantified under the stated experimental conditions. Blank samples were measured together with samples with concentrations of the analyte at the expected LOD. The signals of the blank and the analyte samples were compared and expressed as a signal-to-noise ratio. The LLQ is the lowest concentration of the analyte in the sample that can be measured with acceptable accuracy and precision under the stated experimental conditions. The signals of the blank samples were compared with the signals from samples which contain known low concentrations of the analyte. Next, a signal to noise ratio at which the analyte can be reliable quantified (3:1 for LOD and 10:1 for LLQ) was determined for plasma matrix. The procedure for LOD and LLQ was repeated 6 times.

\section{Stability}

Stability of RAM and TEL in plasma samples was studied at three different stability conditions such as short-term, freeze-thaw, and long-term stability which were examined by replicate analysis of the low, medium and high concentration samples spiked in plasma matrices. Short term stability was carried out by keeping replicate samples for approximately $6 \mathrm{~h}$. Freeze-thaw stability of samples was obtained over three freeze-thaw cycles, by thawing at room temperature for $2-3 \mathrm{~h}$ and refrozen for $12 \mathrm{~h}$ for each cycle. Longterm stability of drugs in animal plasma was tested after storage at$80^{\circ} \mathrm{C}$ for $30 \mathrm{~d}$. For each concentration and storage condition, six replicates were analyzed in one set. 


\section{Ruggedness and robustness}

To evaluate ruggedness and robustness of methods, effects of variations of certain variables were investigated like $\mathrm{pH}$, analyst, material, flow-rate, column temperature, mobile phase composition, and detection wavelength and extraction solutions. Results are shown in results and discussion [6].

\section{Statistical analysis}

The mean plasma concentration was calculated using simple statistical application. Paired t-test was applied as a statistical tool for evaluation of differences in saliva vs plasma concentrations. A ttest dependent correlation was used to compare correlation coefficient. Samples above the normal range and below the detection limit for HPLC assay were not included in the analysis.

\section{RESULTS AND DISCUSSION}

\section{Selection of $\lambda_{\max }$}

The absorption of a standard solution of TEL and RAM was recorded in the wavelength range of $210-370 \mathrm{~nm}$ against methanol as blank. As showed in fig. 3 and $4, \lambda_{\max }$ was found to be 270 [7] and $273 \mathrm{~nm}$ respectively for TEL and RAM.

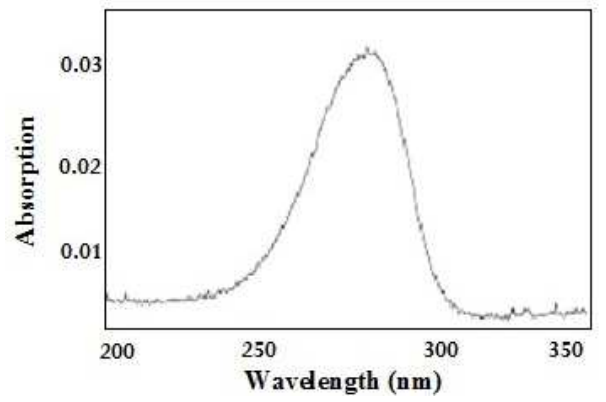

Fig. 3: Ultraviolet spectroscopy of TEL at $270 \mathrm{~nm}$

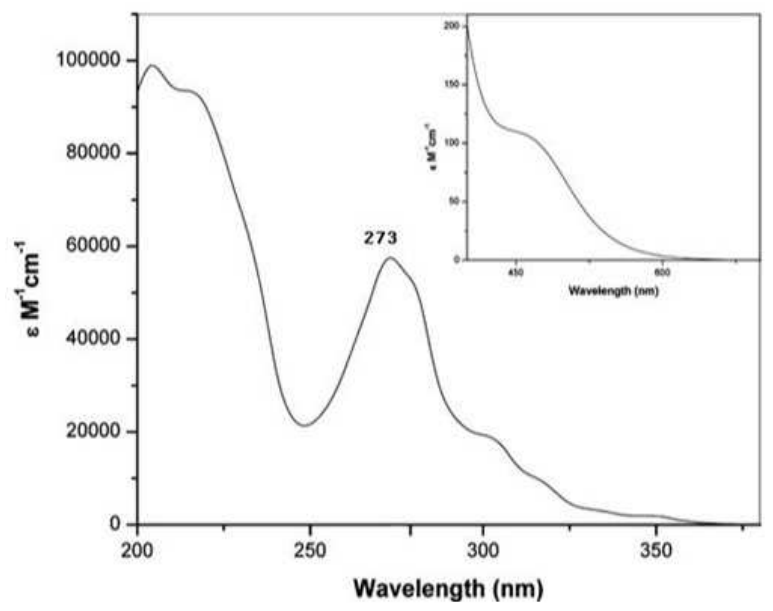

Fig. 4: Ultraviolet spectroscopy of RAM at $273 \mathrm{~nm}$

\section{Method development}

Based on the solubility profile, pKa, polarity of a drug molecule and other factors like stability etc, various mobile phase combinations, flow rate, $\mathrm{pH}$ and $\lambda_{\max }$ was selected and samples were run for optimum selection of chromatographic parameters to develop and validate the RP-HPLC method for estimation of TEL and RAM in biological matrices like plasma [8]. All samples and solutions were prepared using solvent system (based on solubility profile) and biological matrix (extracted as discussed in methodology) as per the FDA guidelines for bioanalytical method development and validation. A detail account of various sets of chromatographic conditions (using a C18 column) for method development of TEL and RAM in Plasma is mentioned in table 1 and 2. The chromatograms for TEL [9] and RAM in Plasma are represented from fig. 5 and 6 respectively.

Table 1: Method development of TEL in plasma matrix using RP-HPLC

\begin{tabular}{|c|c|c|c|c|c|c|}
\hline \multirow[t]{3}{*}{ Conditions } & \multicolumn{2}{|c|}{ Mobile phase (A: B) } & \multirow{3}{*}{$\begin{array}{l}\lambda_{\max } \\
(\mathrm{nm})\end{array}$} & \multirow{3}{*}{$\begin{array}{l}\text { Flow rate } \\
\mathrm{ml} / \mathrm{min}\end{array}$} & \multirow{3}{*}{$\begin{array}{l}\text { Compound } \\
\text { fig. no. }\end{array}$} & \multirow[t]{3}{*}{ Remarks } \\
\hline & A & B & & & & \\
\hline & ACN* $^{*}$ & $\mathrm{MeOH}^{* *}$ & & & & \\
\hline 1 & 40 & 60 & 273 & 0.5 & $5(\mathrm{~A})$ & Peak was not well separated, LONG run time \\
\hline 2 & 35 & 65 & 272 & 0.8 & $5(\mathrm{~B})$ & $\begin{array}{l}\text { Peak shape for TEL was good but detector saturation was observed } \\
\text { for TEL at } 1 \mu \mathrm{l} \text {. }\end{array}$ \\
\hline 3 & 30 & 70 & 270 & 1.0 & $5(\mathrm{C})$ & Peak shape for TEL was good and retention time for TEL was 6.3. \\
\hline 4 & 20 & 80 & 270 & 1.2 & $5(D)$ & Animal Plasma Spiked with I. S. \\
\hline 5 & 15 & 85 & 270 & 1.4 & $5(\mathrm{E})$ & Mobile Phase \\
\hline 6 & 10 & 90 & 270 & 1.4 & $5(\mathrm{~F})$ & blank plasma \\
\hline
\end{tabular}

${ }^{*} \mathrm{ACN}=$ Acetonitrile, ${ }^{* *} \mathrm{MeOH}=$ Methanol

Table 2: Method development of RAM in plasma matrix using RP-HPLC

\begin{tabular}{|c|c|c|c|c|c|c|}
\hline \multirow[t]{3}{*}{ Conditions } & \multicolumn{2}{|c|}{ Mobile phase (A: B) } & \multirow[t]{3}{*}{$\lambda_{\max }$} & \multirow{3}{*}{$\begin{array}{l}\text { Flow rate } \\
\mathrm{ml} / \mathrm{min} .\end{array}$} & \multirow{3}{*}{$\begin{array}{l}\text { Compound } \\
\text { fig. no. }\end{array}$} & \multirow[t]{3}{*}{ Remarks } \\
\hline & $\mathbf{A}$ & $\mathbf{B}$ & & & & \\
\hline & MeOH* & $\mathrm{H}_{2} \mathrm{O}^{* *}$ & & & & \\
\hline 1 & 90 & 10 & 273 & 0.5 & $6(\mathrm{~A})$ & Prolonged run time, peaks not resolved \\
\hline 2 & 85 & 15 & 273 & 0.8 & $6(\mathrm{~B})$ & Peaks Overlap \\
\hline 3 & 80 & 20 & 273 & 1.0 & $6(C)$ & Tailing and long run time \\
\hline 4 & 75 & 25 & 273 & 1.2 & $6(D)$ & Slight Tailing \\
\hline 5 & 72 & 28 & 273 & 1.0 & $6(\mathrm{E})$ & Peak shape for RAM were good \\
\hline 6 & 70 & 30 & 273 & 1.2 & $6(\mathrm{~F})$ & Mobile Phase \\
\hline
\end{tabular}

${ }^{* *} \mathrm{MeOH}=$ Methanol, ${ }^{* *} \mathrm{H}_{2} \mathrm{O}=$ water with dilute orthophodphoric acid (pH 4.5)

\section{Method validation}

TEL and naproxen (I. S.) and RAM and vasartan (I. S.) were well separated from the co-eluted components and there were no interferences from the endogenous material which was observed at retention time of both TEL and RAM with their respective I. S. Peaks of samples were of good quality and completely resolved from plasma components. The retention time of I. S. and TEL were found 
to be 4.0 and $6.3 \mathrm{~min}$, respectively, and the runtime was $8 \mathrm{~min}$ Results revealed that the developed method was highly selective for the matrices like plasma as shown in fig. 5(C) and 6(E) for TEL and RAM respectively.
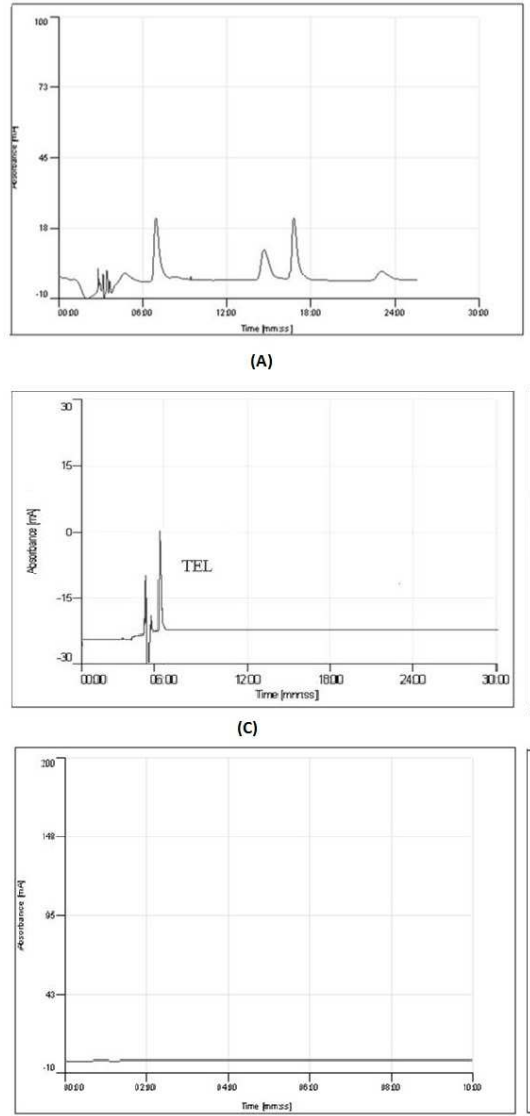
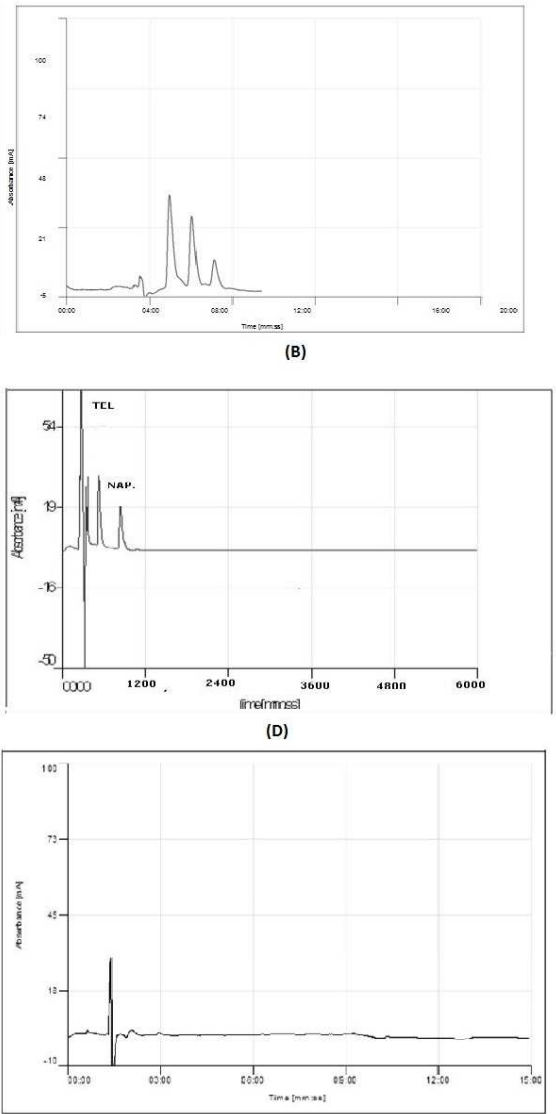

(F)

Fig. 5: HPLC chromatogram for TEL and I. S. with different chromatographic conditions (as shown in table 1) (A) condition 1; (B) condition 2; (C) condition 3, TEL spiked in blank animal plasma; (D) Condition 4; (E) Condition 5; (F) Condition 6 for mobile phase
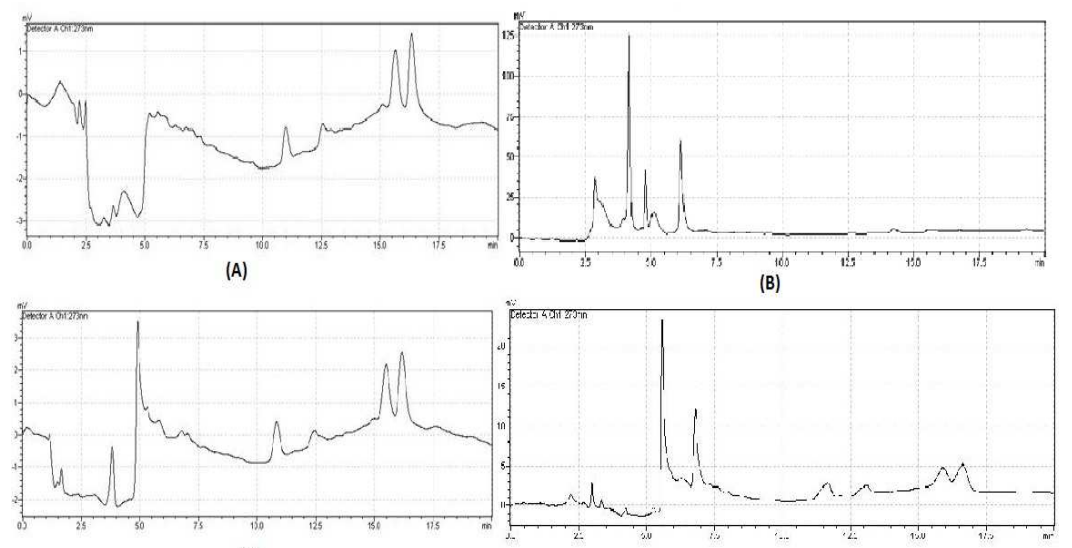

(c)

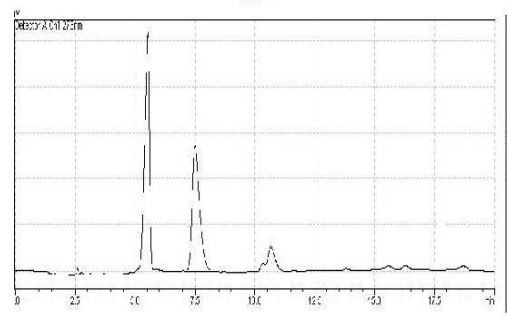

(E)

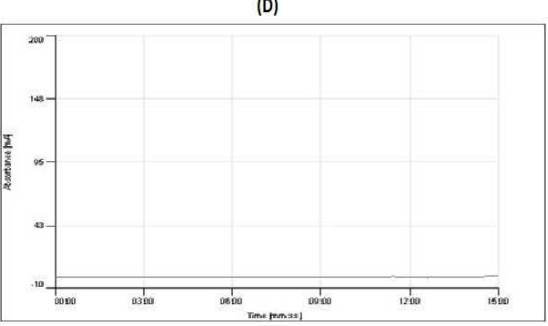

(F)

Fig. 6: HPLC chromatogram of RAM and I. S. with different chromatographic conditions (as shown in table 1) (A) Condition 1; (B) Condition 2; (C) Condition 3; (D) Condition 4; (E) Condition 5 animal plasma spiked with I. S.; (F) Condition 6 for mobile phase 
The accuracy and precision studies of TEL and RAM have been carried out by analyzing five determinations per concentration [three concentrations representing the entire range of the standard curve]. The results of accuracy were found to be in the range of $88.60 \%$ to $97.41 \%$ (table 3, 4). Therefore, based on the recovery data the estimation of related compounds that are prescribed in this report has been demonstrated to be accurate for the intended purpose and is adequate for routine analysis. The precision study has been done for intra and inters day variations for three consecutive days.

The precision of the proposed method for RAM, expressed as \% CV was determined by the analysis of three different concentrations. The intra-day precision was assessed from the results of five replicate analysis of quality control samples on the same day. The inter-day precision was determined from the same quality control samples analyzed on three consecutive days.

Table 3: Validation of TEL

\begin{tabular}{|c|c|c|c|c|c|c|c|}
\hline \multirow{2}{*}{$\begin{array}{l}\text { S. } \\
\text { No. }\end{array}$} & \multirow{2}{*}{$\begin{array}{l}\text { Criteria } \\
\text { Added } \\
\text { conc. }\end{array}$} & \multicolumn{3}{|l|}{ Within-run } & \multicolumn{3}{|l|}{ Between run } \\
\hline & & $\begin{array}{l}\text { Nominal conc. } \\
(\text { mean } \pm \text { SD) }\end{array}$ & $\begin{array}{l}\text { Accuracy } \\
\text { (\%) }\end{array}$ & $\begin{array}{l}\text { Precision (\% } \\
\text { RSD) }\end{array}$ & $\begin{array}{l}\text { Nominal conc. } \\
(\text { mean } \pm S D)\end{array}$ & $\begin{array}{l}\text { Accuracy } \\
\text { (\%) }\end{array}$ & $\begin{array}{l}\% \\
\mathrm{CV}\end{array}$ \\
\hline TEL & & & & & & & \\
\hline 1 & 5 & $004.43 \pm 00.12$ & 88.60 & 2.70 & $004.39 \pm 00.23$ & 87.80 & 5.26 \\
\hline 2 & 25 & $022.81 \pm 00.99$ & 91.24 & 4.34 & $023.46 \pm 01.29$ & 93.84 & 5.49 \\
\hline 3 & 125 & $113.17 \pm 05.71$ & 90.54 & 5.04 & $109.81 \pm 06.02$ & 87.85 & 5.48 \\
\hline
\end{tabular}

$*_{n}=6$

Table 4: Validation of RAM

\begin{tabular}{llll}
\hline Day & Time & \% Concentration (mean \pm SD) & RSD \\
\hline $\mathbf{1}$ & T1 & $91.23 \pm 2.9673$ & 2.8541 \\
& T2 & $93.72 \pm 4.1569$ & 4.2789 \\
$\mathbf{2}$ & T3 & $97.41 \pm 2.2195$ & 2.2786 \\
& T1 & $93.72 \pm 4.0325$ & 4.1677 \\
& T2 & $95.03 \pm 4.4897$ & 4.6212 \\
$\mathbf{3}$ & T3 & $90.20 \pm 1.5486$ & 1.4987 \\
& T1 & $95.13 \pm 2.0031$ & 2.1852 \\
& T2 & $94.87 \pm 4.1025$ & 4.2149 \\
\hline
\end{tabular}

$* \mathrm{n}=6$

The recovery of an analyte is the retention factor obtained from an amount of the analyte added to and extracted from plasma compared to same obtained for the true concentration of the quality control sample. Recovery experiments have been performed by comparing the analytical results for extracted samples at three concentrations as specified in quality control sample analysis with unexpected standards that represent 100\% recovery. Over the concentrations studied, minimum extraction recovery of TEL and IS were found to be 86.96 and $91.40 \%$ respectively. While for RAM and IS were found to be 86.96 and $91.40 \%$ respectively (table 5)

Table 5: Recovery studies of RAM and TEL with their respective IS in animal plasma

\begin{tabular}{|c|c|c|c|c|c|}
\hline S. No. & Drugs & Amount added & Amount recovered (mean \pm SD) & \% recovery & $\% \mathrm{CV}$ \\
\hline 1 & RAM & 1 & $0.98 \pm 00.01$ & 98.00 & 1.02 \\
\hline 2 & & 2 & $2.01 \pm 0.03$ & 100.5 & 1.49 \\
\hline 3 & & 3 & $2.99 \pm 0.04$ & 99.67 & 1.34 \\
\hline 4 & TEL & 5 & $04.49 \pm 00.23$ & 90.00 & 5.1 \\
\hline 5 & & 25 & $21.87 \pm 01.13$ & 87.48 & 5.2 \\
\hline 6 & & 125 & $108.70 \pm 04.98$ & 86.96 & 4.6 \\
\hline
\end{tabular}

$*_{n}=6$

The linearity of the method was evaluated by processing fivepoint calibration curves. Good linearity was observed over the concentration range of $5-250 \mathrm{ng} / \mathrm{ml}$ and $0.5-5 \mu \mathrm{g} / \mathrm{ml}$ for TEL and RAM respectively. The peak area versus concentrations of drugs was plotted and a linear least square regression analysis was conducted to determine the slope, intercept and correlation coefficient $\left(\mathrm{r}^{2}\right)$ to demonstrate the linearity of the method. The correlation coefficient $\left(\mathrm{r}^{2}\right)$ in all cases was found to be $>0.9987$ indicating a functional linear relationship between concentrations of analyte and the areas under the peak. The limit of detection and limit of quantitation was established by evaluating the signal to noise ratio of $3: 1$ and 10:1, respectively. The signals of the blank samples were compared with the samples containing known low concentrations of the analyte. The LOD (S: N; 3:1) and LOQ (S: N; 10:1) was found to be $2.01 \pm .05 ; 4.88 \pm 0.10$ and 0.11 and 0.25 for TEL and RAM respectively on the basis of a signal to noise ratio.
The ruggedness of the method at various parameters was found to be $\pm 1.94 \%$ and $\pm 1.02 \%$ for TEL and RAM respectively. For robustness of TEL and RAM, evident from ANOVA statistical test: the calculated F value was found to be less than tabulated $F$ value $(0.768637<4.06618$ and $0.6876<4.06618$ respectively) indicated that method was robust enough for the analysis of TEL and RAM within the specified range of deviation in the experimental conditions. The low values of \% RSD for each of the drug proposed that during all deliberate variations, middle-quality control (MQC) was not affected and it was in accordance with that of actual. Hence, the newly developed analytical method was considered to be robust [10].

\section{Stability}

The stability of the drug-spiked at three QC levels evaluated for: short-term (6 h), freeze-thaw (3 cycles), and long-term (30 d). The results showed that the TEL was stable in animal plasma for about one month when stored in the frozen state $\left(-80^{\circ} \mathrm{C}\right)$. The accuracy 
was greater than $89 \%$ and $87 \%$ in all cases for TEL and RAM respectively and within the predefined standard limits for bioanalytical methods (table 6).

\section{Clinical application for monitoring plasma levels of TEL}

The developed and validated method was applied for routine therapeutic monitoring of TEL and RAM in the plasma of hypertensive animals. The therapeutic range of both drugs was determined in plasma samples.
In the present study, therapeutic range in which most of the animals $(\mathrm{n}=12)$ were stable was $5-250 \mathrm{ng} / \mathrm{ml}$. A typical chromatogram obtained from animal plasma sample was shown in fig. 5(C). Amongst the 6 outliers, 3 animals were below range $(<5 \mathrm{ng} / \mathrm{ml})$ and 3 animals above range $(>250 \mathrm{ng} / \mathrm{ml})$. The outliers were either therapeutically compromised or showed an adverse effect associated with high concentration of drug-like sweating, flushing, fever, malaise, tinnitus, earache, and toothache etc.

Table 6: Stability studies of TEL and RAM in animal plasma

\begin{tabular}{|c|c|c|c|c|}
\hline DRUG & Conc. $(\mathrm{ng} / \mathrm{ml})$ & Plasma conc. (mean \pm SD) $)^{*}$ & \% Accuracy & CV \% \\
\hline \multicolumn{5}{|c|}{ Short term stability (6h) RT } \\
\hline \multirow[t]{3}{*}{ TEL } & 5 & $004.47 \pm 00.21$ & 89.00 & 4.70 \\
\hline & 25 & $023.21 \pm 01.12$ & 92.84 & 5.21 \\
\hline & 125 & $115.40 \pm 07.74$ & 92.32 & 6.70 \\
\hline \multicolumn{5}{|c|}{ Long term stability $\left(-80^{\circ} \mathrm{C}, 1 \mathrm{Mo}\right)$} \\
\hline & 5 & $04.43 \pm 00.38$ & 89.00 & 8.58 \\
\hline & 25 & $23.17 \pm 02.14$ & 92.68 & 9.24 \\
\hline & 125 & $107.11 \pm 09.31$ & 85.68 & 8.69 \\
\hline \multicolumn{5}{|c|}{ Freeze and thaw cycles } \\
\hline & 5 & $04.52 \pm 00.22$ & 90.40 & 4.87 \\
\hline & 25 & $23.81 \pm 00.82$ & 95.24 & 3.46 \\
\hline & 125 & $111.43 \pm 06.64$ & 89.14 & 5.96 \\
\hline \multicolumn{5}{|c|}{ Short term stability (6hr) RT } \\
\hline \multirow[t]{3}{*}{ RAM } & 1 & $1.09 \pm 0.1105$ & 107 & 3.44 \\
\hline & 2 & $1.98 \pm 0.7821$ & 101.30 & 7.72 \\
\hline & 3 & $2.67 \pm 1.2251$ & 89.56 & 4.55 \\
\hline \multicolumn{5}{|c|}{ Long term stability $\left(-80^{\circ} \mathrm{C}, 1 \mathrm{Mo}\right)$} \\
\hline & 1 & $1.85 \pm 0.1319$ & 97.00 & 4.53 \\
\hline & 2 & $02.17 \pm 0.6718$ & 97.70 & 6.87 \\
\hline & 3 & $2.92 \pm 1.4121$ & 88.40 & 5.32 \\
\hline \multicolumn{5}{|c|}{ Freeze and thaw cycles } \\
\hline & 1 & $0.977 \pm 00.22$ & 90.40 & 4.87 \\
\hline & 2 & $2.67 \pm 0.2123$ & 89.00 & 7.95 \\
\hline & 3 & $2.79 \pm 1.4413$ & 93.00 & 5.16 \\
\hline
\end{tabular}

$*_{n}=6$

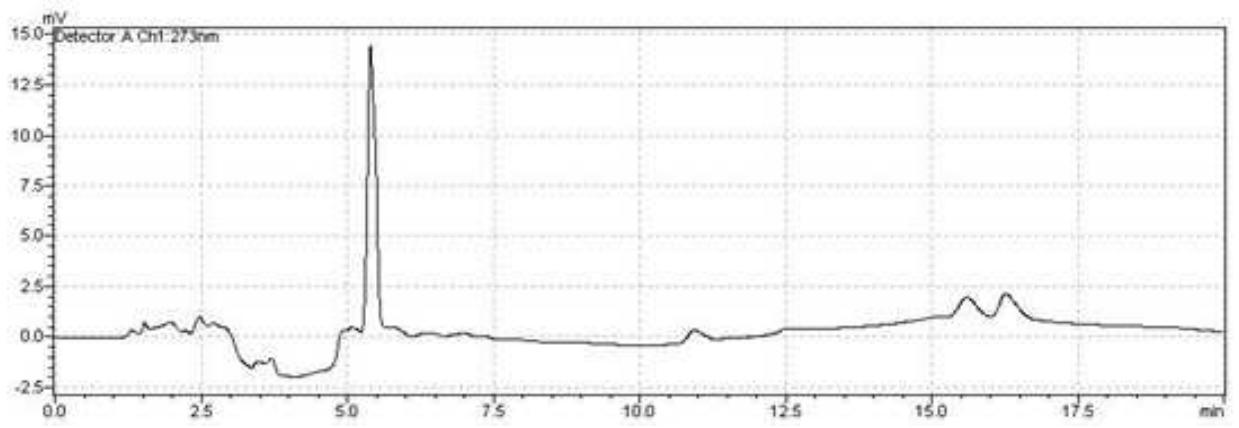

Fig. 7: Typical chromatogram of RAM in animal plasma

\section{Clinical application for monitoring plasma levels of RAM}

Therapeutic range in which most of the animals $(n=29)$ were stable was found to be $0.5-5 \mu \mathrm{g} / \mathrm{ml}$. A typical chromatogram obtained from animal plasma sample was shown in fig. 7.

Amongst the 10 outliers, three animals were below range $(<0.4 \mu \mathrm{g} / \mathrm{ml})$ and seven animals above range $(>25.6 \mu \mathrm{g} / \mathrm{ml})$. The outliers were either therapeutically compromised or showed an adverse effect associated with high concentration of drug-like hypotension, decrease urination, dry irritating cough, and high $\mathrm{K}^{+}$level. The regimen of these animals was changed later as per their clinical condition.

\section{Therapeutic drug monitoring of TEL and RAM}

Various mobile phase combination, flow rate, $\mathrm{pH}$ and $\lambda_{\max }$ was selected and samples were run for optimum selection of chromatographic parameters to develop and validate RP-HPLC method (as per the FDA guidelines for bioanalytical method development and validation) for estimation of TEL and RAM in a biological matrix like plasma.

\section{Method validation}

TEL, RAM and I. S. were not well separated from the co-eluted components and there was interference from an endogenous material which was observed at a retention time of TEL, RAM and IS. There were no identifiable peaks, and not completely resolved from plasma components.

\section{Clinical application of the method}

The method was applied for routine estimation of RAM and TEL in hypertensive animals. A total of 10 animals were enrolled in the 
study and further 6 included as per inclusion-exclusion criteria, out of which four animals completed the study. The method we applied for routine clinical monitoring of TEL and RAM in animals (fig. 8) showed that there were no specific peaks of the desired drugs.

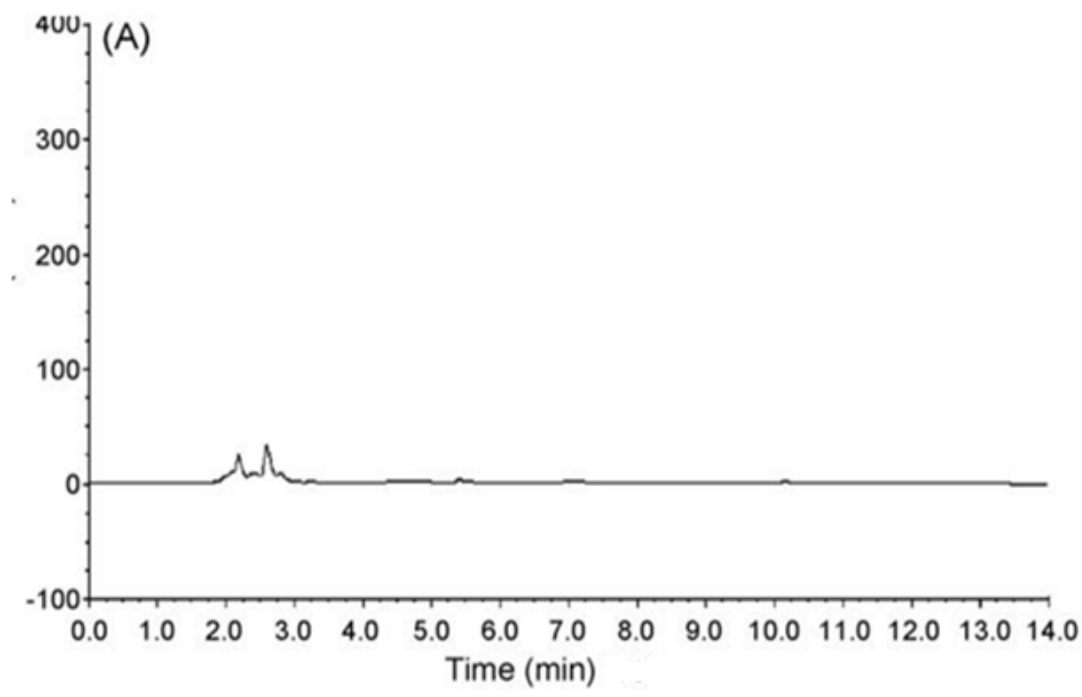

Fig. 8: Validation of developed method in animal plasma (TEL $40 \mathrm{mg}+\mathrm{RAM} 5 \mathrm{mg}$ )

But by monitoring these animals it was observed that combination therapy was not significantly better than alone TEL and RAM. Surprisingly, despite a reduction in systolic blood pressure of 2-3 $\mathrm{mmHg}$ in the combination therapy as compared with the RAM group. The animals were clinically stable with a definite therapeutic level; below which the condition got worse and above which there was no therapeutic benefit but adverse effects precipitated. Developed methods reported the least interference of endogenous substances from plasma for separate estimation of TEL and RAM. Extraction recovery studies revealed excellent efficiency of extraction and precision studies showed that method was accurate and precise. We found a low LOD and LOQ which have proven sensitivity of the method to detect minute quantities of TEL and better accuracy and precision. Thus developed HPLC method was found to be more accurate, precise, sensitive, selective and reproducible.

Although sometimes useful for proteinuria reduction and in the treatment of symptomatic animals with heart failure, the combination of an ACE inhibitor and an angiotensin receptor blocker (ARB) is not recommended for the treatment of hypertension. ACE/ARB combinations produce little additional $\mathrm{BP}$ reduction compared with monotherapy with either agent alone. In the ongoing TEL Alone and in Combination with RAM global endpoint trial, animals receiving the ACE inhibitor/ARB combination showed no improvement in cardiovascular endpoints despite additional blood pressure (BP) reduction averaging 2.4/1.4 $\mathrm{mmHg}$. There were also more side effects with the combination than with individual agents. These combinations are classified as less effective.

\section{CONCLUSION}

In the present study, the attempt has been undertaken to develop the most simple, economical, sensitive and accurate analytical HPLC method for the simultaneous estimation of these drugs without their prior separation. The method gives good resolution between both the compounds with a short analysis time $(<10 \mathrm{~min})$. The method was validated and found to be simple, sensitive, accurate and precise. The developed methods have found to have the least interference of endogenous substances from plasma for separate estimation of TEL and RAM. Therefore, the proposed method can be used for routine analysis of RAM and TEL in their combined dosage form.

\section{ACKNOWLEDGEMENT}

Authors thank to Pranveer Singh Institute of Technology for providing facilities for research work.

\section{AUTHORS CONTRIBUTIONS}

All the author have contributed equally

\section{CONFLICT OF INTERESTS}

There is no conflict of interest

\section{REFERENCES}

1. Tripathi KD. Medical pharmacology. 6th ed. New Delhi: Jaypee Brothers; 2008. p. 501-8.

2. The United States Pharmacopoeia 29, National Formulary 24 Asian Edition. Rockville MD. United States Pharmacopoeial Convention, Inc; 2006. p. 501-8.

3. Rao KV, Vijaya Kumari K, Bhanuprakash I, Prabhakar G, Begum J. Determination of ramipril in pharmaceutical dosage forms by reversed-phase liquid chromatography. Asian J Chem 2006;18:788-92.

4. Belal F, Al-Zaagi IA, Gadkariem EA, Abounassif MA. A stabilityindicating LC method for the simultaneous determination of ramipril and hydrochlorothiazide in dosage forms. J Pharm Biomed Anal 2001;24:335-42.

5. Rao RN, Sen S, Nagaraju P, Reddy VS, Radha Krishnamurthy P, Udaybhaskar S, et al. HPLC determination of Telmisartan in bulk and pharmaceutical formulations. Asian J Chem 2006;18:775-82.

6. ICH, Q2B-Validation of Analytical Procedures: Methodology, International Conference on Harmonization; 1996.

7. Salama I. Simultaneous HPLC-UV analysis of telmisartan and hydrochlorothiazide in human plasma. Bull Faculty Pharmacy Cairo University 2011;49:19-24.

8. Rajoriya V, Soni A, Kashaw V. Method development and validation of fast dissolving tablet of ramipril by HPLC method. Int J Pharm Pharm Sci 2016;8:174-8.

9. Madhukar A, Kannappan N, Mahendra Kumar CB. Analytical method development and validation for the determination of hydrochlorothiazide, amlodipine besylate and telmisartan hydrochloride in the multicomponent tablet dosage form and in biorelevant media (FASSIF) by RP-HPLC techniques. Int J Pharm Pharma Sci 2015;7:218-25.

10. Mahesh M, Kumanan R, Jayaveera KN. Isocratic RP-HPLC-UV method development and validation for the simultaneous estimation of hydrochlorothiazide and ramipril in tablet dosage form and bulk drug. Int J Curr Pharm Res 2011;3:119-23. 